\title{
Industrial Engineering from the Vision of a Portable Automated Model
}

\author{
Lourdes Medina, PhD. ${ }^{1}$, Ricardo Silva, Bs. ${ }^{1}$, Zachary Soto, Bs. ${ }^{1}$ \\ ${ }^{1}$ Universidad de Puerto Rico, Puerto Rico, lourdes.medina@upr.edu, ricardo.silva@upr.edu, zachary.soto@upr.edu,
}

\begin{abstract}
It can be generally stated that the industrial engineering (IE) field branches out to a broad spectrum of aspects, not limiting itself to one focus in the industry. In a world that is constantly advancing technologically, IE is key in allowing the advancement of production, whether in a manufacturing or service industry, in order to keep up with the everyday increasing demand. From the more social standpoints dealing more with people, such as supervision to the more technical, say economics, quality and production forecasting, IE impacts the field in many ways. These different aspects can all be seen and represented through one of the more increasingly common practices in the field, automation. Accordingly, this paper shows how we can teach IE from the vision of a portable automated model (PAM). Through the development of $P A M$, many aspects and branches of IE can be displayed to show the versatility of $I E$ roles and purposes and its different impacts in the industry. Using a small-scale model, different examples of cost and accounting, economic analysis, lean, design, layout, production planning controls, operations, simulation, quality and even human resource management can be displayed to show the importance of this engineering field.
\end{abstract}

Keywords- Engineering Education, Industrial Engineering, Automation

Digital Object Identifier (DOI): http://dx.doi.org/10.18687/LACCEI2015.1.1.072

ISBN: 13 978-0-9822896-8-6

ISSN: 2414-6668

$1^{\text {th }}$ LACCEI Annual International Conference: “Engineering Education Facing the Grand Challenges, What Are We Doing?” July 29-31, 2015, Santo Domingo, Dominican Republic ISBN: 13 978-0-9822896-8-6

ISSN: $2414-6668$

DOI: http://dx.doi.org/10.18687/LACCEI2015.1.1.072 


\section{Industrial Engineering from the Vision of a Portable Automated Model}

\begin{abstract}
It can be generally stated that the industrial engineering (IE) field branches out to a broad spectrum of aspects, not limiting itself to one focus in the industry. In a world that is constantly advancing technologically, $I E$ is key in allowing the advancement of production, whether in a manufacturing or service industry, in order to keep up with the everyday increasing demand. From the more social standpoints dealing more with people, such as supervision to the more technical, say economics, quality and production forecasting, IE impacts the field in many ways. These different aspects can all be seen and represented through one of the more increasingly common practices in the field, automation. Accordingly, this paper shows how we can teach IE from the vision of a portable automated model (PAM). Through the development of PAM, many aspects and branches of IE can be displayed to show the versatility of IE roles and purposes and its different impacts in the industry. Using a small-scale model, different examples of cost and accounting, economic analysis, lean, design, layout, production planning controls, operations, simulation, quality and even human resource management can be displayed to show the importance of this engineering field.

Keywords- Engineering Education, Industrial Engineering, Automation
\end{abstract}

\section{INTRODUCTION}

The Institute of Industrial Engineers (IIE) defines industrial engineering (IE) as a study "concerned with the design, improvement and installation of integrated systems of people, materials, equipment and energy. It draws upon specialized knowledge and skill in the mathematical, physical and social sciences together with the principles and methods of engineering analysis and design to specify, predict and evaluate the results to be obtained from such systems." [1]. It can be generally stated that the IE field branches out to a broad spectrum of aspects, not limiting itself to one focus in the industry. In a world that is constantly advancing technologically, IE is key in allowing the advancement of production, whether in a manufacturing or service industry, in order to keep up with the everyday increasing demand. From the more social standpoints dealing more with people, such as supervision to the more technical, say economics, quality and production forecasting, IE impacts the field in many ways. These different aspects can all be seen and represented through one of the more increasingly common practices in the field, automation.
Automation is the use of control systems to control a process, more often seen in manufacturing, which in turn, reduces human intervention in the same. Automation has existed since the 1910's in Ford's Assembly Line for their famous car production [2]. By applying mechanical, hydraulic and pneumatic components, many tasks in the process were simplified, allowing the reduction of human intervention yet increasing production. Note, an assembly line can be composed of both human intervention and automation, be it that operators serve as loaders or intermediaries within the process or as skilled technicians assigned to tools. While job reductions quickly come to mind when the word automation is mentioned, the scenario is not as black and white. Automation should be looked at as a method of increasing production by making a process more efficient, but this also allows for better use of the previous resources to help expand production. In a business perspective, it is always recommended to use resources efficiently, automation allows for a greater distribution of such when creating the transformation from a more manual scenario.

Through the development of a portable automated model (PAM) prototype, many aspects and branches of IE can be displayed to show the versatility of IE roles and purposes and its different impacts in the industry. Using a small scale model, different examples of cost and accounting, economic analysis, lean, design, layout, production planning controls, operations, simulation, quality and even human resource management can be displayed to show the importance of this engineering field.

\section{BACKGROUND}

PAM initially started off as a regular course project for Real Time Process Control and was later chosen to be adapted into a portable form. Making the model portable makes it easier to transport to schools to allow students to see the impact of IE through the model. PAM represents an automated assembly process initiating from obtaining the inventory to final packaging after quality inspection. For the purpose of giving it a theme for school demonstrations, it was decided that the PAM is part of a process in a candy factory, making one of three different assortments. 
First, a box must be placed in Station 1, which decides what assembly will be made. The box is then pushed to the assembly line in front of Station 2, which assembles the pieces onto the box. Once in the box, the same is pulled along the line Station 3 with an approximate $50 \%$ chance to either go straight into the final station or go to inspection Station 4. At Station 4 a quality test is made to declare whether or not the assembly came out defective, which is declared by a green light if it's a pass, or a red light and a buzzer if it turns out to be defective.

\section{A. Station 1: Tray Classification}

Station 1 as seen in Figure 1 is the initiation of the process that decides which of the two different assemblies will be made depending on the box placed in the station. The station has a limit switch to detect the placement of a box, and an inductive sensor that detects the main difference between both boxes. If the metal is detected for two seconds, assembly 2 (semi circles) is initiated, else, assembly one commences. A bar then pushes the box onto the main assembly line where an electromagnet holds the box in place.

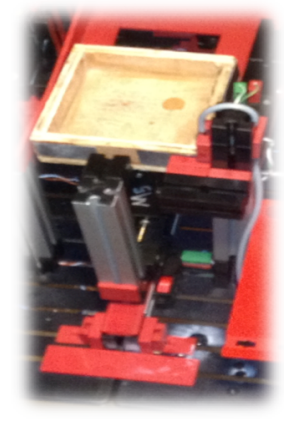

Figure 1: Station 1

\section{B. Station 2: Parts Assembly}

The parts assembly (Figure 2) is composed of two different feeders for parts to create two different assemblies.

\section{1) Assembly 1}

When assembly 1 is initiated, a device similar to a ski lift is activated which is automatically loaded with inventory dropped from an inclined ramp. As the circles are picked up, they are then dropped onto a ramp fitted with a proximity sensor that upon detecting the object halts the "ski-lift" conveyor. The material slides off the ramp and onto the assembly platform where a mechanical arm positions it to the center of the platform and pushes it onto the box previously at station 1 . When the mechanical arm returns back to its initial position, this process is repeated once more for a second piece in order to complete the assembly. Note, this assembly is done directly onto the box.

\section{2) Assembly 2}

Assembly two initiates two conveyors that move the semicircles directly onto the assembly platform. The conveyors are fitted with two phototransistors on either side towards the end, both of which are in an activated state by a light bulb placed between the two separate conveyors. When either phototransistor detects that the light has been blocked by a piece, it halts the conveyor until the other conveyor does the same. Once both pieces are in place, meaning both conveyors have stopped, the conveyor is initiated for the duration of one second, allowing both pieces onto the platform, but not permitting any others to pass the phototransistors. Keep in mind that even if a following piece ends up in front of the phototransistor, all this does is not activate the conveyor in the next run, serving as a poka-yoke.

When both semicircles are on the assembly platform, the mechanical arm pushes them together and onto the box on the assembly line. Once the mechanical arm returns to its initial position, the assembly process is concluded.

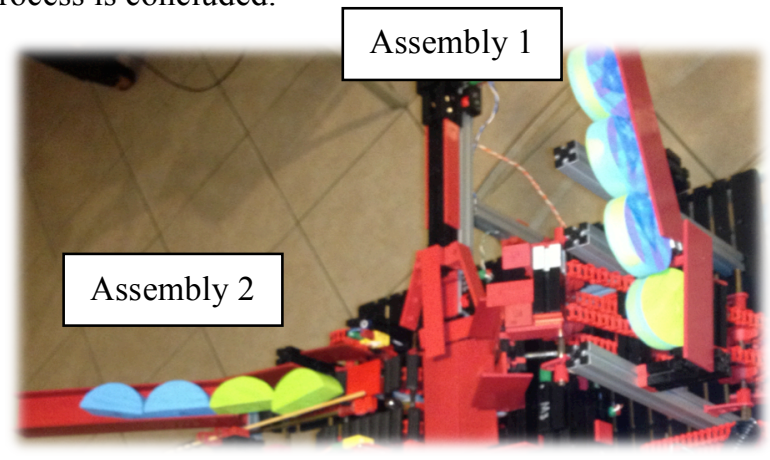

Figure 2: Parts assembly

\section{Station 3: Inspection Decision}

Inspection decision is merely in the programing where, if and only if, the condition, set by timers outside of the stages, is met while the limit switch is being pressed in the assembly line, the mechanical arm extends out to the conveyor to deliver the box.

\section{Station 4: Inspection}

The inspection station as seen in Figure 3 is composed of a vertical mechanical arm with 4 limit switches protruding at the bottom which upon being activated extends down to secure contact on all four corners of the box, the one way to secure not only that the assembly is in the box, but that both are 
properly placed. And electromagnet attached to a motor on the bottom then moves the box onto the final conveyor.

\section{E. Station 5: Final Station}

Final station (Figure 4) is simply the movement of the box onto the shipping platform. Whether from the inspection station or the main line, the conveyors retrieve the box and move it onto the platform where the bulbs let operators know if the piece is defective or not.

\section{F. Hand Made Parts}

Although the majority of the parts and tools used for the PAM were bought, several components were hand crafted due to the specific needs and purpose of the project. The PAM makes two different types of assemblies, both of which create a wooden cylinder. The difference between both parts is in the way they are cut. Assembly 1 (Figure 5) consists of the cylinder cut horizontally while assembly 2 (Figure 6) consists of a vertical cut. Using thin woodcuts, two different sets of boxes were made for the two different assemblies. Boxes 1 and 2 can be seen in Figure 7. While box type 1 is used for the flat circles and box type 2 is used for the semi-circles, the only difference between the two is that box type 2 has a metal contact on its corner. The boxes are made of thin woodcuts, sanded down and glued with industrial glue along with metal squares in each upper corner and the sides cut from a metal sheet. By putting metal in each corner, the direction in which the box is placed is irrelevant, serving as a Poke-

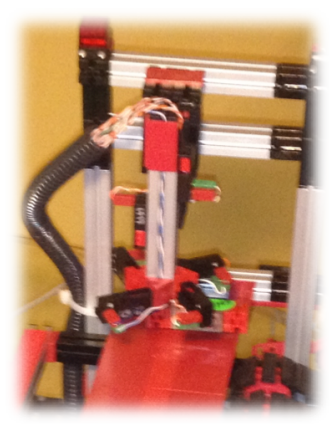
Yoke.

\section{Figure 3: Inspection station} Figure 4: Final station

Figure 5: Assembly 1
Figure 6: Assembly 1

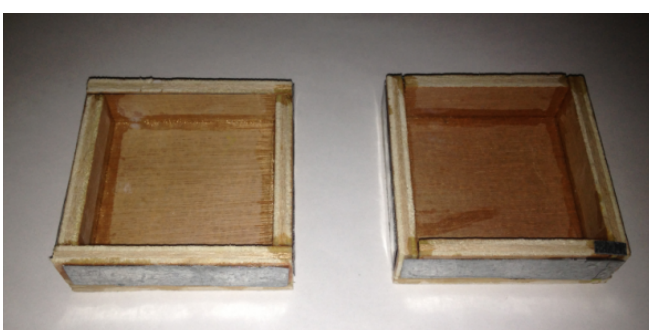

Figure 6: Box type 1 and 2

\section{METHODOLOGY}

The methodology and process previously mentioned to create the PAM is summarized in Figure 8. As is visible, it is all divulged inside one square-titled "Trial Runs". Trial runs are the core activity in successfully creating the model. Many time random components will fail in the model, and trial runs allows the catching of those mistakes beforehand. The implementation of the flipping device and soldering the circuitry are bordered in red because they were the most challenging parts of this process. As for the flipping device, the difficulty of creating that component without consuming more space turned out to be a more tedious task than the resulting reward, hence the solution with two conveyors and the applied phototransistors was decided. The resulting model can be seen in Figure 9.
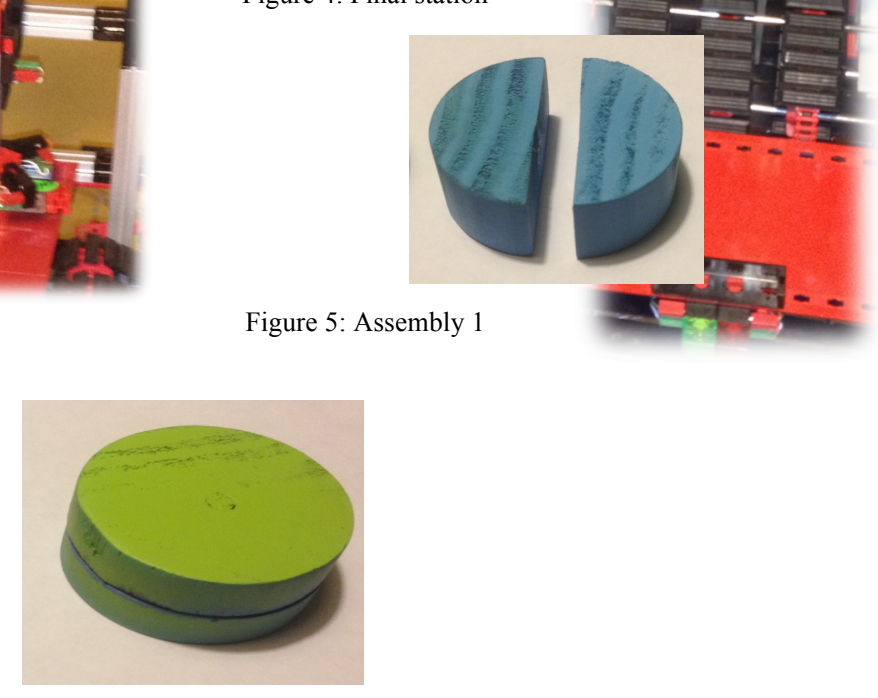

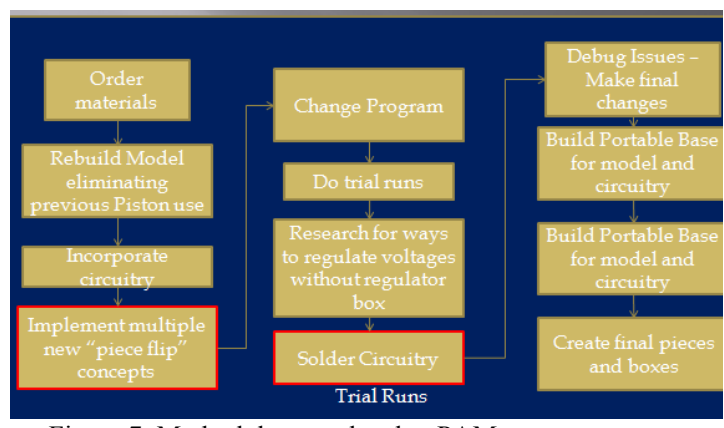

Figure 7: Methodology to develop PAM

13th LACCEI Annual International Conference: "Engineering Education Facing the Grand Challenges, What Are We Doing?” July 29-31, 2015, Santo Domingo, Dominican Republic 


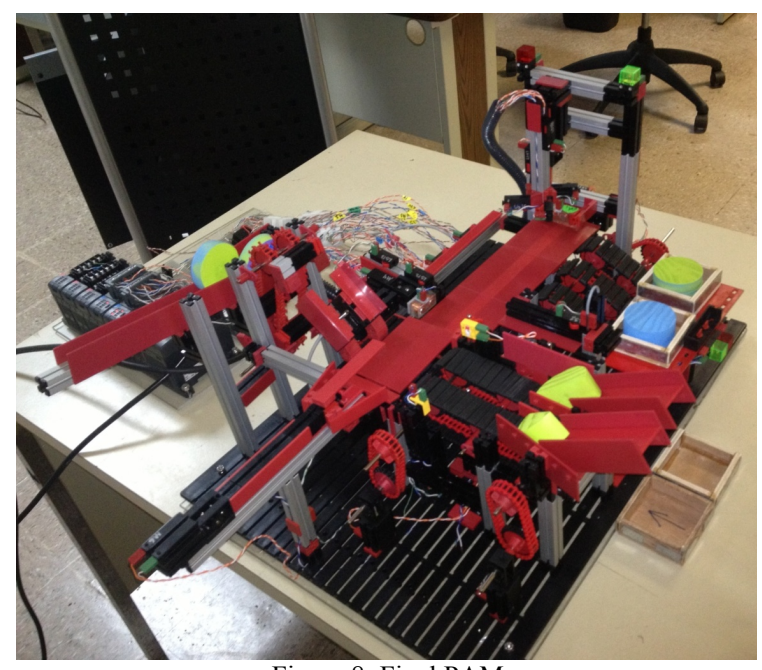

Figure 8: Final PAM

In order to have the PAM fully serve its purpose, a good presentation had to be created. In order to execute properly, there is one factor that must be taken into consideration beforehand: The audience. The audience in the case of this investigation is students from intermediate to high school grades. Having that in mind, the approach to present took this into consideration. First decision that was made was the presentation method. While posters were considered, the well-trusted power point presentations (PPPs) were chosen. First and foremost, PPPs allow for more control on what is being presented to the students in the form of slides. With a poster, a presenter can talk about a topic while a student is reading something else. The most important thing in doing these presentations is having the students' focus. They should be entertained while absorbing as much information as they are being fed. PPPs also allow bigger and better display, allowing bigger groups of students to equally see what is going on, securing that no one gets left out. Also, PPP's allow for multimedia components, these being videos, music and more pictures. The ever-changing dynamic of a PPP captures a student's attention when a new picture or video pops up.

A study done states that, "One common belief is that the typical student's attention span is about 10 to 15 minutes long. This belief has been reinforced in numerous books and articles on teaching."'[3] The presentations conducted aim to avoid this by engaging the students. By mixing the PAM with the PPP and relatable topics, we secure that the students devote their attention to the presentation.
While the presenters will be college students being educated and molded to present to professionals, wearing business attire and being serious on a professional level, this is unnecessary in this case. These are young students whose interests are all about what they find appealing. It is for this reason that one must make a connection with them. When presenting, make the students laugh by incorporating a sense of humor into the presentation. A polo representing the university with some nice jeans and sneakers still makes you presentable, but the students will feel less intimidated and more forward, a very important characteristic when they are wanted to be engaged.

Even when applying this, the full attention of the students is not always secured. This is why the presenters must constantly pay attention to the student's attention spans. If the presenter can tell a student is dozing off, or their attention has gone to a completely different area of the room, change up the routine, and find new and different ways to incorporate them. Include goodies and prizes that they can win if they answer a few questions, or maybe talk to their teacher before the presentation to see if they have any topics relating to their class that can be incorporated with the PAM. For example, in the presentation previously done, the students that were presented to where from a math course, so a math problem was introduced in the presentation where they had to calculate how much was the pay per hour given the hours and direct labor costs incorporated on the PAM. This will keep the students on edge, knowing that the class also depends on their attention of the presentation.

It is suggested that the actual PAM be demonstrated towards the end of the presentation, this allows for the students' attention to be "jumpstarted" by having them all come to the front of the class and see the model in action. At this moment they tend to ask questions referenced to what they just learned, and vice versa allow the presenters to ask questions and secure that the students understood various key components.

\section{INDUSTRIAL ENGINEERING THROUGH THE PAM}

In the project's presentation, relationships were made between the different topics and courses of the University of Puerto Rico - Mayaguez Campus' IE Department and PAM. While the upcoming topics focus on those themes, when presenting, different

13th LACCEI Annual International Conference: "Engineering Education Facing the Grand Challenges, What Are We Doing?” July 29-31, 2015, Santo Domingo, Dominican Republic 
examples of different situations were discussed to secure the important connection the students need between what we want them to learn and what they already know.

Before talking about the topics, the students were asked what they knew already about IE, and while the answers were generally broad, a positive response was always feedback to secure constant participation. Upon explain that IEs make processes more efficient, they were asked if they had eaten at Krispy Kremes. The barrage of happy shouts and hands in the air was the sign that we had their attention and complete interests, and we immediately used that opportunity to explain how the Krispy Kreme's donut process was a perfect example of an automated and efficient process with many different IE components.

Another eye-catching example was a slide in the presentation full of well renowned companies like Boeing, Texas Instruments and General Electric amongst many others. The students were asked how many of those companies they heard of, and immediately started shouting out names and the products that they themselves had seen or used. They were then informed that every single one of the companies on that slide had hired many of our colleagues from our graduating class, and we related that by telling them to just picture that their friends sitting in the room were the ones going to those companies, something which could be a reality if they study IE.

\section{A. Leadership/People}

It's ironic to think that in the topic of automation, people would be a viable topic for an IE, but in reality, it can be considered the most important of all. In an ever-changing manufacturing world, people still play the role as the tide changer. When it comes to creating change, those affected by the process can either serve as resistance or as a great boost of momentum. IEs undergo several courses in the field of psychology and social sciences with the purpose of increasing skills to manage people in the workplace dynamic. When any type of project involving change is in action, an IE needs to be able to not only address the proposal to upper management, but also have operators and those impacted by the project to jump on the bandwagon in order to secure a smoother outcome and obtain better results. It is clearly seen in the industry that when operators become more positively involved in a project they provide ideas that hit critical points due to their experience in the process.

\section{B. Human Resource Planning}

For an automated process, managing the different levels of roles is extremely important. Before executing anything, people are the first obstacle. While for upper management, costs and benefits with the adequate payback period in a project proposal can be enough, convincing those directly affected by the automation can prove a more difficult task. The human resource planning course prepares students for similar situations, helping students prepare to manage situations, dealing with topics such as: (1) finding ways to motivate and get people to join along, (2) making ethical and unbiased decisions, (3) leading teams to come up with new solutions, (4) performance management, and (5) resolving conflicts in the workplace.

Resolving conflicts in the workplace is crucial to handling resistance. An IE that does not know how to manage and work well with others will be held back in professional growth, compared to those that can. Even after the process is automated, the IE still needs to analyze the situation and communication is key. Do the tool technicians need more training? Is everything running as efficiently as planned? Talking with the teams can secure perfection.

In the presentation, two slides were included: one with happy faces and one with frustration faces. The objective was to show that not always things go well and stress can be a part of a process, but managing that stress and working well with others is something all IEs learn. While conveying that message, the slide also served as way to incorporate humor, resulting in several laughs from the students followed by nods of understanding. This is a perfect example of the previously mentioned need to engage the students with different methods.

\section{Lean}

In this day and age, the most heard of methodology in the field of IE, or in the industry for that matter, is lean manufacturing. Lean, in its most essential definition, is the elimination of waste. When undertaking a role in lean, there is a specific goal, to make more using less. While there are many workshops that offer the ever so famous lean belts, these do not match the depth that IE provides. Lean can be seen as the tool, while industrial courses provide a detailed 'how to' on using the tool, the statistical and theoretical reasoning plus all the added perks and gadgets that make it more effective. For the presentation, a slide showing a before and after of the

13th LACCEI Annual International Conference: "Engineering Education Facing the Grand Challenges, What Are We Doing?” July 29-31, 2015, Santo Domingo, Dominican Republic 
circuits was used to demonstrate lean from the $5 \mathrm{~S}$ perspective, showing the students that by implementing the new soldered circuit, new improvements or fixes were easier to apply.

\section{Work Measurements}

When dealing with any type of process, it is important to know the average time it takes for a process to run in order to know its production capacity and the necessary resources in order to meet standards. These two factors are broad in respect to all the other things they affect. In terms of production capacity we ask ourselves: (1) Are there any tasks creating bottlenecks in the process? (2) What are my critical points in the process?, and (3) Are tools or stations being used effectively? When dealing with resources, work measurements help answer: (1) What is the necessary inventory? and (2) How many operators are required?

Applying work measurement studies and creating balanced lines of production secure efficient use of resources. More importantly, work measurements also take into consideration human and mechanical factors. Time allowances are created to take into consideration fatigue and other factors that affect human employees, creating more realistic time standards instead of assuming a robotic pace that would otherwise be unachievable by a person working 8 hours a day. In terms of machines, allowances are also incorporated to take into account down times and other aspects such as cool downs or inspections. These realistic times allow for a better estimate to what a line can produce without the expense of operator health and machine lifetime.

In terms of the automated process, a work measurement study can be applied to determine the rate at which the model is making the product. Going into a more detailed analysis of the process, balancing can be done to attempt changes in the process itself. Can I run two different loads simultaneously? Maybe three? Depending on the times of the different stations, it can be inferred at which points new loads can be introduced into the system without interfering with the ones being currently processed. At what rate will I have to load new inventory? Should any of the motors be sped up to allow more production, or will it just consume more energy at no true gain? All these questions can be answered through the use of work measurement.

In the presentation, a slide (Figure 10) following the work measurements had a picture of the PAM with the different process times. Having previously explained what a process bottleneck was, the students were asked to find the bottleneck, all of which they immediately did, but interestingly enough, one student surprised the presenting team by stating "So if we lower that time, does that mean that the new bottleneck is the station with $13 \mathrm{~s}$ because it would be the slowest?" This unexpected response showed that not only did the student learn the term, but also the point of it in practice.

\section{E. Work Systems Design}

As previously mentioned, process automation does not necessarily mean that there will be no human intervention, and when the case arises for a human operator to interact in a station, their safety and wellbeing is more important than production. Work Systems Design deals directly with ergonomics. Also known as human factors, ergonomics "is the scientific discipline concerned with the understanding of the interactions among humans and other elements of a system, and the profession that applies theoretical principles, data and methods to design in order to optimize human wellbeing and overall system performance."[5] Some of these factors are physical, cognitive and organizational, all of which have effects leading to stress.

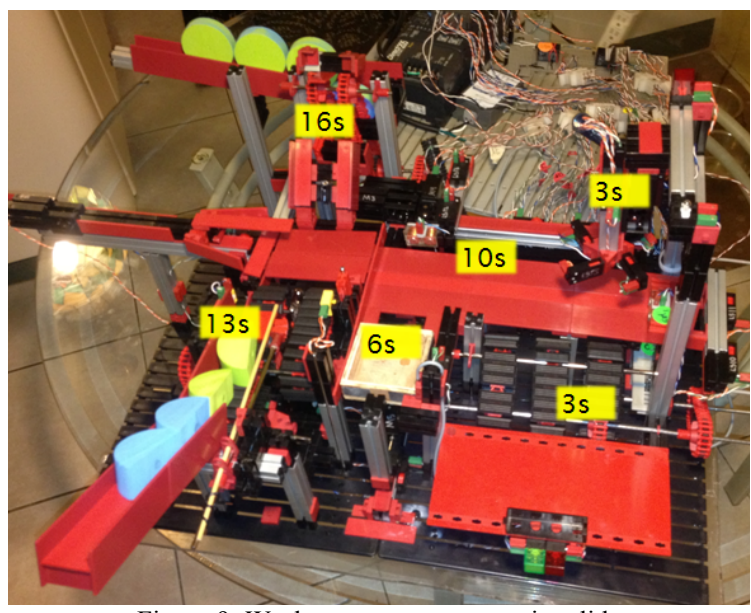

Figure 9: Work measurement exercise slide

The course itself deals with the design of work stations that decrease repetitive movements, avoid fatigue from pulling or lifting forces, allow for proper levels of sound, incorporate appropriate lighting and secure a comfortable sitting or standing position, depending on the task. There is an expansive array of multivariable settings for these situations depending on the operator's gender, age, body dimensions,

13th LACCEI Annual International Conference: "Engineering Education Facing the Grand Challenges, What Are We Doing?” July 29-31, 2015, Santo Domingo, Dominican Republic 
assembly parts size and even the station's surface color.

If the process automated model was to be applied to an actual factory floor, when the case arises for loading inventory and unloading the completed assembly, the design would have to make sure that the operators don't have to lift their arms higher than their shoulder height or have to bend over and that the inventory and final assemblies themselves don't weigh more than allowed. Also, the motors utilized would have to be measured in terms of sound to secure adequate levels, and then loading plans would have to be created in order to allow for the operator to take the necessary breaks while simultaneously avoiding the halt of production.

For the presentation they were shown the proper placements of hands when using a mouse and screwdriver, explaining that in the process of building the PAM, health is very important and always making sure we were not injuring ourselves in the process was utmost priority.

\section{F. Layout and Physical Facilities Design}

Layout and Physical Facilities Design is a course that explains the fundamental importance when designing new layouts, these being the process, walking distances, space consumed and carrying costs for materials. The students were demonstrated the three different designs that were made for the model, asked which they though was used and the answers ranged between all three. The students we told they were all correct due to the fact that as a team, the model used the best components of all three designs to make the final result. Upon demonstrating the model students asked why only one assembly platform was used which we used to explain how having only one assembly platform allowed for less space use and in return less costs.

\section{G. Quality Statistics and Control}

As IEs, we seek perfection in processes along with customer satisfaction. Quality Statistics and control serves to minimize defects in a process and find the causes of the defects, resulting in lines being what is referred to as control. The main example from the PAM is the inspection station itself, which helps in avoiding any defects going out of the factory by alerting with the light and buzzing sound. Quality is very important, but the degree to which it is ranges on the product being produced. When manufacturing toys, the allowed defects are very different from a company that manufactures airplanes. It is necessary to point out how IE's also affect people's lives and it's the reason many take great pride in what they do. When manufacturing medical devices, there should be no tolerance for defects because due to any error, others can die. This type of example captures student's attention in a more impacting way.

\section{H. Economic Analysis, Accounting and Costs Analysis}

The different economical courses in IE serve to show the versatility of IE. These courses are the main source to answer the golden question in the industry: Is a company making or losing money?

While economic analysis deals more along the lines of fluctuating costs from past, present and future, accounting and cost analysis deal more with current state of affairs. The amount of money the company is generating and the break-even point are two components discussed to the students. With the break-even point the students were explained that if the PAM was to be applied to a company, the same would have to calculate how long it would take for the process itself to generate the amount of money it costs.

Money is a term all children and teens understand. While these courses are more analytical, presenting them to students is simpler when talking about large quantities of money. A strong talking point was the amount of money that some colleagues and ourselves had generated for companies through our projects, ranging from the 100 thousands to the millions. The surprised look of students asking "did you really make that as students?" proves it to be an effect technique.

\section{Production Planning and Control}

Production planning and control are a hand in hand analysis with lean. When doing improvements or simply altering production levels, the topics learned in these courses really cement the results by making effective plans on inventory levels and such. Material and inventory cost money, and costs need to be reduced. Production planning and control aid in reducing these costs by deciding how much and when a company should order material. Future demands are calculated in this type of analysis that helps decide whether or not certain products should be focused on.

The way this topic was presented took advantage of the PAM's different assemblies. As previously mentioned, there are three assortments. Assembly

13th LACCEI Annual International Conference: "Engineering Education Facing the Grand Challenges, What Are We Doing?” July 29-31, 2015, Santo Domingo, Dominican Republic 
two makes two different candies, the blue and green, while assembly one makes the mystery flavor candy which is the resulting excess of candy mix left when exchanging materials in the machine. As it turns out, the clients like the flat circle candy much more, hence in the PAM there is more inventory of these. Simple relationships like these aid in having the students see how one product can be ordered more than another and in return, how production plans are made.

\section{J. Simulation and Programming}

Simulations are necessary when identifying capabilities of lines, before or after changes are made. Through the use of ARENA, the simulation course shows how to take advantage of the amazing simulating capabilities of the program and apply them to real world processes. The program allows the inclusion of all the different factors of a process including but not limited to cycle times, resources, stations and queue limits.

In the presentation was highlighted that the simulation course professor participated and aided in the creation of ARENA. This topic is important to bring up in the presentations because it shows the students that the professors we have do and have made impacts on the industry, in this case, a program used worldwide.

Simulation is not the only programming course but also algorithms which shows how to program in VBA, very effective when wanting to do programs for situations like recalling databases and such. As for the PAM, a picture showing the DIRECTSOFT program was included, showing the students another skillset found in the large array of necessary IE skills.

\section{ASSESSMENT}

A short survey (Figure 11) was made to 24 students in order to evaluate the efficiency of teaching IE from the vision of PAM. In a four point likert scale (0nothing, 1- little, 2- regular, 3- a lot) students were asked three questions. The first question was to evaluate the students' IE knowledge prior to the presentation, meanwhile the second question asked the IE knowledge gained after the presentation. The last question was in regards to automation, where students were asked if the automated model was useful in helping them understand the different IE concepts. Table 1 summarizes the results from the survey in percentage of students that selected each option. From this feedback we interpret that the presentation was a success given that $75 \%$ had little or no knowledge about IE, 91\% expressed to have gained regular or a lot of knowledge about IE and $91 \%$ indicated that incorporating an automated model was regular or a lot useful. Student comments were also very amusing: "I'm interested in Industrial Engineering." "I liked the presentation and I learned a lot on Industrial Engineering." "It was useful to understand it and it's a really cool thing to study."

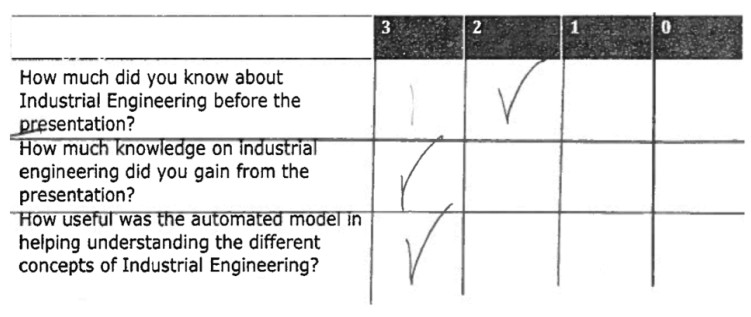

Figure 10: Survey example

TABLE I

PERCENTAGE OF STUDENTS SELECTING EACH OPTION

\begin{tabular}{|l|r|r|r|r|}
\hline & 3 & 2 & 1 & 0 \\
\hline Question 1 & $4.17 \%$ & $20.83 \%$ & $37.50 \%$ & $37.50 \%$ \\
\hline Question 2 & $29.17 \%$ & $62.50 \%$ & $8.33 \%$ & $0.00 \%$ \\
\hline Question 3 & $54.17 \%$ & $37.50 \%$ & $8.33 \%$ & $0.00 \%$ \\
\hline
\end{tabular}

\section{CONCLUSION}

While the PAM is an effective tool to demonstrate the different components and topics of IE, it is not enough just by itself. The incorporation of a power point presentation is excellent in controlling what the students are seeing and allowing several multimedia components to draw the attention and assure student involvement in the presentation itself. After presenting to the school and receiving the students' feedback, the success of the PAM was un-doubtable. The students were entertained the whole way through, and at times when their attention lapsed, they were engaged by questions or activities. At the end and throughout the presentation they were all asking questions and making comments relating to previously discussed topics and it was clear that they had a much better understanding of what IEs do. As stated, their interest is the main goal of the presentation, upon getting their interest, they pay more attention to what is said. IEs have work and examples available anywhere that there is a process, and this must be taken advantage of. Disney's fast pass system, Krispy Kremes donut creation and Boeing's huge $747 \mathrm{~s}$ flying through the air are all talking points that involve IEs. IE is an excellent

13th LACCEI Annual International Conference: "Engineering Education Facing the Grand Challenges, What Are We Doing?” July 29-31, 2015, Santo Domingo, Dominican Republic 
career path, and the impressions of these students confirmed that.

\section{ACKNOWLEDGES}

We would like to thank the College Access Challenge Grant for their support in this project.

\section{REFERENCES}

[1] Institute of Industrial Engineers. (n.d.). Institute of Industrial Engineers. Retrieved December 15, 2013, from http://www.iienet.org
[2] Meyer, S., 2004, "The Degradation of Work Revisited: Workers and Technology in the American Auto Industry, 19002000." Automobile in American Life and Society. http://www.autolife.umd.umich.edu /Labor/L Overview/L Overview3.htm〉

[3] Are You with Me? Measuring Student Attention in the Classroom . (n.d.). The Teaching Center. Retrieved December 13, 2013, from http://teachingcenter.wustl.edu/Journal/Reviews/Pages/studentattention.aspx

[4] International Ergonomics Association. (n.d.). International Ergonomics Association. Retrieved September 8, 2013, from http://www.iea.cc/01_what/What\%20is\%20Ergonomics.html

[5] HFES, Definitions of Human Factors and Ergonomics, Human Factors and Ergonomics Society. http://www.hfes.org/web/educationalresources/hfedefinitionsmain. html

13th LACCEI Annual International Conference: "Engineering Education Facing the Grand Challenges, What Are We Doing?” July 29-31, 2015, Santo Domingo, Dominican Republic 